\title{
Agro-morphological Characterization and Genetic Diversity Analysis of Cotton Germplasm (Gossypium hirsutum L.)
}

\author{
K. Rathinavel* \\ Central Institute for Cotton Research, Regional Station, Coimbatore-641003, India \\ *Corresponding author
}

\begin{tabular}{l} 
K e y w or d s \\
Cotton, Germplasm, \\
Genetic diversity, \\
Correlation, \\
Principal \\
component analysis, \\
Clustering \\
\hline Article Info \\
\hline $\begin{array}{l}\text { Accepted: } \\
\text { 15 January } 2019 \\
\text { Available Online: } \\
\text { 10 February } 2019\end{array}$ \\
\hline
\end{tabular}

Keywords

Cotton, Germplasm, Genetic diversity,

Correlation,

Principal

component analysis,

Clustering

Article Info

Accepted:

Available Online

\section{A B S T R A C T}

The working group of $G$. hirsutum germplasm accessions was characterized for Distinctiveness, Uniformity and Stability testing. Subsequent analysis of data was done to study the genetic diversity available among the accessions using principal component and clustering of 320 cotton germplasm. Under field and laboratory, 26 qualitative traits and 14 quantitative traits were recorded. There is no variation observed for gossypol glands, anther filament colour, male sterility, boll bearing habit and boll opening. Higher coefficient of variation was recorded for vigour index, seed cotton yield/row, germination percentage, seed cotton yield/plant and fibre strength. In the Pearson's correlation, the number of bolls per plant, number of sympodia, seed cotton yield per row, fibre elongation showed positive significant correlation with seed cotton yield per plant. These traits can be directly used as selection criteria for yield improvement in cotton. In the principal component analysis, five principal components (PCs) extracted had Eigenvalue $>1$ and contributed $76.80 \%$ of variations among the cotton germplasm. The clustering using UPGMA showed 12 distinct clusters. Based on these, an accession of a particular group or clusters may be selected for exploitation of its yield potential and fibre quality.

\section{Introduction}

Cotton, the most important commercial fibre crop, plays a major role in the socio-economic and political world. Globally it is cultivated in about 31.11 million hectares (Anon, 2016) in all continents except Antartica. The world production is 22.4 million metric tonnes (Anon, 2016). Cotton is the king of fibre crops and key money-maker in Indian agriculture sector. India has the largest area of global cotton cultivation accounting 11.8 million hectares by surpassing China during
2015. Its contribution to the global cotton production is $27 \%$. Cotton plays a key role in the Indian economy in terms of income and employment generation in agricultural and industrial sectors. India has the distinction of having the largest area under cotton cultivation in the world ranging between 11.9 million hectares to 12.8 million hectares and constituting about $38 \%$ to $41 \%$ of the world area under cotton cultivation. The yield per hectare ranges from 504 to $566 \mathrm{kgs}$ per hectare, is however still low against the world average of about 701 to $766 \mathrm{kgs}$ per hectare 
(Anon, 2016). Low productivity could be attributed broadly to an improper selection of genotypes and lack of crop management practices. To overcome these, one such approach is genetic enhancement and production potential of cultivars. Though all the four species of the genus Gossypium viz., $G$. arboreum, G. herbaceum (old world cotton) G. barbadense and G. hirsutum (new world cotton) is cultivated, G. hirsutum takes the lion share owing to its fibre quality and high yield potential and hence, the data of $G$. hirsutum working germplasm collections characterised morphologically for Distinctiveness, Uniformity and Stability (DUS) analysis were utilised for genetic diversity analysis. Diversity among germplasm is of great concern to a perspective crop improvement programme, as it should be to cotton producers. This depends on the creation of genetic variation between parental lines for a unique gene combination, necessary for a new superior cultivar. Extensive use of closely-related cultivars by producers resulted in vulnerability to pests and diseases. Plant breeders often make use of germplasm lines to develop improved genotypes for the upcoming environmental conditions that completely outclass the previous genotypes in terms of performance (Khan et al., 2015). The variability for economic attributes in the given germplasm is vital for gratifying exploitation following selection and breeding (Sajjad, et al., 2011).

Therefore, proper knowledge of genetic variability and further study on this is the paramount milestone in the understanding of interspecies as well as intra-species resultant crop performance and yield improvement. Genetic variation based upon morphological and agronomic attributes has been exploited in cotton for victorious future breeding (Ahmad et al., 2012), which requires very high level of perfection because they affect with different environmental conditions and hence, characterization of these traits need fully matured plants prior to tagging and identification (Sundar et al., 2014).

In crop improvement programme, crop yield will be the first and foremost criteria to be vouched, a complex biometrical trait and its genetic analysis are rather difficult. Seed cotton yield is a resultant product of all its component traits and it could be enhanced by exploiting positive influence of yield components. Multivariate biometrical techniques like principle component analysis (PCA), Correlation Analysis and Clustering method have been frequently used to explore genetic diversity among genotypes and its direct and indirect effects (Brown-Guedira et al., 2000). Genetic variation of morphological traits estimated through principal component analysis has led to the recognition of phenotypic variability in cotton (Sarvanan et al., 2006; Esmail et al., 2008; Li et al., 2008). Keeping this in view the present study was executed to explore genetically divergent genotypes utilising the DUS morphological traits with desirable correlated agronomic attributes.

\section{Materials and Methods}

The experimental material for the present study consisted of 320 G. hirsutum germplasm accessions raised in Augmented Block Design at Central Institute for Cotton Research, Regional Station, Coimbatore. Seeds of each line were spaced $45 \mathrm{~cm}$ within the row and $90 \mathrm{~cm}$ apart from the other row. Recommended agronomic and plant protection measures were followed from sowing till harvest of the crop.

The data on 26 qualitative and 14 quantitative characters were recorded on the specified growth stage of the cotton plant following National test guidelines for the conduct of Distinctness, Uniformity and Stability (DUS) 
of tetraploid cotton (Gossypium spp.) in India (Plantauthority.Gov.in).

The qualitative traits observed were hypocotyl pigmentation, leaf colour, leaf hairiness, leaf appearance, gossypol glands, leaf nectaries, leaf petiole pigmentation, leaf shape, stem hairiness, stem pigmentation, bract type, petal colour, petal spot, position of stigma, anther filament colouration, pollen colour, male sterility, boll bearing habit, boll colour, boll shape, boll surface, boll prominence of tip, boll opening, seed fuzz, seed fuzz colour and fibre colour in ten randomly selected plants. The quantitative traits viz., fibre length, fibre strength, fibre fineness, fibre uniformity, fibre elongation were recorded. In addition to above DUS traits, data on ancillary traits such as number of sympodia, number of bolls per plant, seed cotton yield (SCY) per row and seed cotton yield per plant, germination (\%) of resultant seed, seedling root and shoot length $(\mathrm{cm})$, vigour index, dry matter of seedling (mg/10 seedling) were also recorded. The data of qualitative traits were used for collating frequency distribution and clustering, while quantitative traits were used for correlation, PCA and clustering.

Mean values of quantitative traits of individual accession were computed for determining the analysis of variance. Pearson correlation coefficient was worked out for quantitative traits and correlation matrix was prepared for comparison of different traits. Principal component analysis (PCA) on quantitative traits was executed in to find out the relative importance of different traits in capturing the genetic variation. The standardised values were used to perform PCA employing the software Minitab. 15. Score plot was used for visual assessment of components or factors that explain most of the variability in the data. The factors corresponding to PCs were subjected to cluster analysis based on Euclidean distances and clustering using hierarchical clustering. Dissimilarity matrix based on Euclidean distance was calculated using these traits by DARwin 6. Most dissimilar and least dissimilar accessions were identified based on dissimilarity matrix. The hierarchical cluster analysis of pooled data was performed using scores of dissimilarity matrix (Ward, 1963).

\section{Results and Discussion}

\section{Qualitative traits}

Qualitative characters are considered as the most important characters to identify a particular plant variety. They are mostly genetically controlled thus least dependent on the environmental response. Variation was found in 21 out of 26 qualitative traits (Table 1). The traits namely gossypol glands, anther filament colouration, male sterility, boll bearing habit and boll opening were shown no variation between genotypes. The character hypocotyl pigmentation showed no pigmentation in $17 \%$ of accessions and the remaining $83 \%$ were pigmented. Among the accessions, green leaf colour was predominant (182) followed by light green (134), dark red (3) and light red (1). For leaf hairiness, sparsely present in 226 accessions followed by medium (86) and dense (8). In 103 accessions leaf appearance was flat nature, whereas 217 expressed cup shape. Leaf nectaries were observed in all genotypes except American nectariless. Pigmented leaf petiole was observed in 202 accessions were absent in118. The Palmate leaf shape was found in 251 accessions followed by semidigitate (42) and digitate (27). Regarding stem hairiness, sparse states of expression were in 144 accessions followed by medium (130), dense (44) and smooth (2). Stem pigmentation was noted in 250 accessions and the remaining was none pigmented. Normal bract was found in 307 accessions and frego bract in rest of the accessions. Expression of cream 
petal colour was recorded in the higher number of accessions (182) followed by yellow (130) and purple (8). Exerted states of flower stigma were recorded in 191 and embedded in 129 accessions. Four genotypes DCB 348 CYFM 531 B Line7, FM 958 B Line1 DELTAPINE (C J) showed spot in the petal and in rest of accessions, it was absent.

The states of expression of pollen colour were cream, yellow, white, deep yellow and purple in $169,96,30,17$ and in 8 accessions, respectively. Boll colour was noted green in 313 accessions and in seven it was red. Ovate boll shape was found in 244 accessions followed by round (49) and elliptic (27). The smooth boll surface present in 309 accessions and 11 accessions had pitted surface. Regarding prominence of boll tip, 314 accessions had the blunt tip and 6 were pointed. Seed fuzz was found in Medium density states in 227 accessions followed by dense (50), sparse (40) states and 3 accessions produced naked seeds. Seed fuzz colour was grey in the majority (288) of accessions, whereas other states like white (20), Green (8) and Brown (4) were also observed. Cream fibre colour in 293 accessions followed by white (20), Green (4) and Brown (3) respectively were observed.

The trait, pollen colour was observed with higher variation (five states) and traits like leaf colour, stem hairiness, the density of seed fuzz, seed fuzz colour and fibre colour had four states while rest of the traits had three and two states. In cotton, Hosseini (2014), reported that the successful hybrids could be recognised and distinguished by morphological markers such as flower colour, spot position and their colours in petal, fibre colour, seed linter, leaf colour and their shapes. Hence the differential observation of qualitative traits in the present study would be much useful for identifying true hybrids in the crop improvement programme.

\section{Clustering}

The cluster analysis of qualitative traits was done based on Euclidean distances which formed the cluster by unweighted paired group method using the arithmetic average (UPGMA). The cluster analysis was done using DARwin 6 software. The dendrogram drawn out of UPGMA depicted six distinct clusters as is presented in Figure 1. The cluster VI was the largest followed by cluster II, cluster III, I, V and IV. May et al., (1995) reported that cluster analysis identified groups of cotton cultivars those were more closely related.

\section{Quantitative traits}

The basic statistics of various traits studied have shown considerable variability among 320 cotton germplasm (Table 2). The largest variation observed was for vigour index, seed cotton yield/row, germination percentage, seed cotton yield/plant and fibre strength. Comparatively, low variation was observed in the dry matter of seedling, fibre length and fibre fineness.

\section{Correlation}

Pearson's correlation (r) is a measure of the strength of association between the two characters. The correlation co-efficient among all characters related to seed cotton yield per plant were estimated and the results are presented in Table 3. Seed cotton yield per plant has significant positive correlation with number of bolls per plant (0.706), number of sympodia (0.465), fibre length (0.430), dry matter of seedlings (0.410), seed cotton yield per row (0.325), fibre elongation (0.248) and negatively correlated with fibre uniformity (0.322). A similar result of the association of seed cotton yield with a number of sympodia was reported by Khan et al., (2015), Salahuddin et al., (2010) and Soomro et al., 
(2008). Morphological traits like sympodia are very important in the cotton plant because sympodia are positively correlated with yield and manage the seed cotton yield (Khan et al., 2011). Therefore it may be concluded that criteria of selection based on a number of sympodia/plant will be helpful for increasing plant yield. Khan et al., 2015, Ahsan et al., (2011), Bibi et al., (2011) and Hussain et al., (2010) also found a positive significant association of a number of bolls per plant with seed cotton yield per plant. Hence, selection of progenies based on this trait will be useful in yield improvement in cotton. Shahzad et al., 2015 recorded positive association of seed cotton yield with a number of bolls, sympodial branches and fibre length. Regarding inter correlation, germination percentage had significant positive correlation with vigour index and seed cotton yield per row; root length significantly correlated with vigour index and shoot length and negatively correlated with fibre elongation. The trait shoot length exhibited significant positive inter correlation with vigour index; Dry matter of seedling has positive inter correlation with the number of bolls per plant, fibre length and fibre elongation and negatively inter correlated with fibre uniformity. The traits like the number of bolls per plant, fibre strength, fibre elongation and the number of sympodia had positive inter correlation with fibre length and negative with fibre uniformity and fibre fineness. Fibre strength had the positive association with the number of bolls per plant and fibre elongation and negative correlation with fibre fineness and fibre uniformity.

\section{Principal component analysis}

Principal component analysis (PCA) clearly indicates the genetic variation of the germplasm. It measures the important characters which have a greater impact on the total variables and each coefficient of proper vectors indicated the degree of contribution of every original variable with which each principal component is associated (Sanni et al., 2008). To find out the independent impact of all the characters under study principal component analysis was conducted.

The five principal components (PCs) extracted had eigenvalue $>1$ and contributed $76.80 \%$ of the variation among the cotton germplasm (Table 4). The first principal component accounted for more than $28.90 \%$ of the total variation. Number of sympodia per plant (0.447), fibre elongation (0.433), seed cotton yield per plant $(0.321)$, boll number per plant (0.319) and fibre strength (0.402) were the variables possibly contributed in this component, among them fibre strength has the negative contribution. It is evident that the PCA1 has identified yield components and fibre quality traits possessing positive and negative contribution to the variables. The above-indicated result is similar to that of the results of correlation analysis. These findings are in the line with Taohua and Yichun, 1993 and Shakeel et al., (2015). The second principal component accounted for $17.9 \%$ of the total variation. Characters highly and positively correlated were vigour index (0.595), root length (0.539) and shoot length (0.502). The third principal component accounted for $13.60 \%$ of the total variation. This component consists of boll number per plant (-0.484), seed cotton yield per row (0.459 ) and seed cotton yield per plant (0.352). Thus the third component registered negative contribution of the variables. It was determined to set cut off limit for the coefficients of the proper vectors (Raji, 2002), According to this criterion, coefficients greater than 0.3 (regardless the direction positive or negative) as having a large enough effect to be considered important, while traits having a coefficient less than 0.3 were considered not to have important effects on the overall variation observed in the present study. 


\section{Biplot}

The Biplot of the principal component of cotton genotypes revealed that closely located genotypes on the graph are perceived as alike when rated on given attributes (Figure 2). Farthest the distance from point of origin more diversified will be the genotypes and vice versa. Figure 2 showed that most cotton genotypes in present investigation situated close to each other on the graph indicating narrow genetic background of cotton genotypes. This might be because of extensive breeding for a limited number of traits. Genotypes such as in MEADE 9030D, 86-1A-1, KH- 113, UA- BK- 4-84, IC 671(SEL), G-COT-100(VISHNU) and XDPI 6317 clogged very near to each other and as well as very close to the point of origin, hence of less breeding value and less diversified. On the other hand, genotypes which clogged at the vertex of the polygon are farthest from point of origin hence more diversified and of high breeding value. The genotypes viz., Buri 0394, UPA (57) -17, EL 958, 70 H 452, B-581290, MDH 90, 6288, RED 5-7, MCU -5 and BJR JK - 97-16 -4 were clogged at the vertex of the polygon. These genotypes are very much useful for future crop improvement programme. This result was in accordance with Khan et al., 2015.

\section{Genotype by trait analysis}

The evaluation and notification of outclassing genotypes for different traits were carried out by using biplot (Figure 2). The accessions viz., 86-1A-1, G-COT-100(VISHNU), BM COT 38 -BLL and AKLA 8 1X TAMCOT SP 21-1 were found in close vicinity with fibre elongation; 24, 252, 81 and 218 were found near fibre length, 249 in close proximity with seed cotton yield per plant, 273 and 126 were clogged near number of bolls per plant, 283 found closer to vigour index, 139 and 148 were found near to root length and shoot length. Hence these genotypes are more related to these traits and will be useful for hybridisation programme. In addition to diversity analysis, the genotypeby-traits (GT) biplot analysis has been used to study the nature of association among the traits, evaluation of genotypes for multiple traits and identification of those genotypes which are superior in certain traits. These genotypes could be the parental lines for a breeding program or for commercial cultivation (Yan and Rajcan, 2002).

\section{Loading biplot}

A biplot constructed through principal components and variables superimposed on the plot as vectors showed that the relative length of the vector represented the relative proportion of the variability in each trait (Fig. $3)$. In the biplot, germplasm accessions which are far away from origin showed more variability with less similarity other varieties. High amount of variability noted for traits like root length, vigour index, shoot length, fibre uniformity, fibre length, number of bolls per plant, seed cotton yield per plant, fibre elongation and fibre strength, whereas traits like fibre fineness, germination percentage, seed cotton yield per row, number of sympodia and dry matter of seedling exhibited the least variability. The quality traits like fibre fineness and fibre uniformity were in a different direction as shown in Figure 3 which was considered undesirable as per the earlier reports of Shakeel et al., (2015).

\section{Score plot}

A score plot emanated out of principal components of the cotton accessions depicted that the accessions those were close together were perceived as being similar when rated based on the variables. Thus accessions representing serial number 38 and 59; 22 and 
26; 62 and 27; 99 and 93; 310 and 320; 5 and 95 were very close to each other from the perspective of both PC1 and PC2 respectively. The accessions representing serial number 134, 172, 225, 60, 151, 7, 1, 61 were rather separated from other accessions. It may be explained that the accession 225 was different from 1 because former lied in positive region and second lied in the negative region of the plot. Likewise, the accession 60 lied opposite to the accession 134 (Fig. 4).

\section{Screen plot}

Screen plot exhibited variance percentage associated with each principal component attained by drawing a graph between eigenvalue and PC numbers. PC1 showed $28.90 \%$ variability followed by $\mathrm{PC} 2$ with $17.90 \%$ having eigenvalues of 4.04 and 2.50, respectively as in Figure 5. Results similar to above was reported by Khan et al., (2015).

Table.1 Frequency distribution of qualitative traits recorded in G. hirsutum accessions of cotton germplasm

\begin{tabular}{|c|c|c|c|c|c|}
\hline S. No. & Variables & Scores & States & No. of genotypes & Frequency $(\%)$ \\
\hline \multirow[t]{2}{*}{1} & \multirow[t]{2}{*}{ Hypocotyl: Pigmentation } & 1 & Absent & 55 & 17.19 \\
\hline & & 9 & Present & 265 & 82.81 \\
\hline \multirow[t]{4}{*}{2} & \multirow[t]{4}{*}{ Leaf: Colour } & 1 & Light green & 134 & 41.88 \\
\hline & & 2 & Green & 182 & 56.88 \\
\hline & & 3 & Light red & 1 & 0.31 \\
\hline & & 4 & Dark red & 3 & 0.94 \\
\hline \multirow[t]{3}{*}{3} & \multirow[t]{3}{*}{ Leaf: Hairiness } & 1 & Sparse & 226 & 70.63 \\
\hline & & 5 & Medium & 86 & 26.88 \\
\hline & & 9 & Dense & 8 & 2.50 \\
\hline \multirow[t]{2}{*}{4} & \multirow[t]{2}{*}{ Leaf: Appearance } & 1 & Cup & 103 & 32.19 \\
\hline & & 2 & Flat & 217 & 67.81 \\
\hline 5 & Leaf: Gossypol glands & 9 & Present & 320 & 100.00 \\
\hline \multirow[t]{2}{*}{6} & \multirow[t]{2}{*}{ Leaf: Nectaries } & 1 & Absent & 1 & 0.31 \\
\hline & & 9 & Present & 319 & 99.69 \\
\hline \multirow[t]{2}{*}{7} & \multirow[t]{2}{*}{ Leaf: Petiole pigmentation } & 1 & Absent & 118 & 36.88 \\
\hline & & 9 & Present & 202 & 63.13 \\
\hline \multirow[t]{3}{*}{8} & \multirow[t]{3}{*}{ Leaf: Shape } & 1 & Palmate & 251 & 78.44 \\
\hline & & 2 & Semi-digitate & 42 & 13.13 \\
\hline & & 3 & Digitate & 27 & 8.44 \\
\hline \multirow[t]{4}{*}{9} & \multirow[t]{4}{*}{ Plant: Stem hairiness } & 1 & Smooth & 2 & 0.63 \\
\hline & & 3 & Sparse & 144 & 45.00 \\
\hline & & 5 & Medium & 130 & 40.63 \\
\hline & & 7 & Dense & 44 & 13.75 \\
\hline \multirow[t]{3}{*}{10} & \multirow[t]{3}{*}{ Plant: Stem pigmentation } & 1 & Absent & 70 & 21.88 \\
\hline & & 9 & Present & 250 & 78.13 \\
\hline & & 5 & $\begin{array}{l}\text { Semi- } \\
\text { spreading }\end{array}$ & 18 & 5.63 \\
\hline \multirow[t]{2}{*}{11} & \multirow[t]{2}{*}{ Bract: Type } & 3 & Normal & 307 & 95.94 \\
\hline & & 5 & Frego & 13 & 4.06 \\
\hline
\end{tabular}


Table.1 Contd..,

\begin{tabular}{|c|c|c|c|c|c|}
\hline \multirow[t]{3}{*}{12} & \multirow[t]{3}{*}{ Flower: Petal colour } & 1 & Cream & 182 & 56.88 \\
\hline & & 2 & Yellow & 130 & 40.63 \\
\hline & & 4 & Purple & 8 & 2.50 \\
\hline \multirow[t]{2}{*}{13} & \multirow[t]{2}{*}{ Flower: Petal spot } & 1 & Absent & 316 & 98.75 \\
\hline & & 9 & Present & 4 & 1.25 \\
\hline \multirow[t]{2}{*}{14} & \multirow[t]{2}{*}{ Flower: Stigma } & 3 & Embedded & 129 & 40.31 \\
\hline & & 5 & Exerted & 191 & 59.69 \\
\hline 15 & $\begin{array}{ll}\begin{array}{l}\text { Anther } \\
\text { colouration }\end{array} & \text { Filament } \\
\end{array}$ & 1 & Absent & 320 & 100.00 \\
\hline \multirow[t]{5}{*}{16} & \multirow{5}{*}{ Flower: Pollen colour } & 1 & White & 30 & 9.38 \\
\hline & & 2 & Cream & 169 & 52.81 \\
\hline & & 3 & Yellow & 96 & 30.00 \\
\hline & & 4 & Deep yellow & 17 & 5.31 \\
\hline & & 5 & Purple & 8 & 2.50 \\
\hline 17 & Flower: Male Sterility & 1 & Absent & 320 & 100.00 \\
\hline 18 & Boll: Bearing habit & 1 & Solitary & 320 & 100.00 \\
\hline \multirow[t]{2}{*}{19} & \multirow[t]{2}{*}{ Boll: Colour } & 3 & Green & 313 & 97.81 \\
\hline & & 5 & Red & 7 & 2.19 \\
\hline \multirow[t]{3}{*}{20} & \multirow[t]{3}{*}{ Boll: Shape } & 3 & Round & 49 & 15.31 \\
\hline & & 5 & Ovate & 244 & 76.25 \\
\hline & & 7 & Elliptic & 27 & 8.44 \\
\hline \multirow[t]{2}{*}{21} & \multirow[t]{2}{*}{ Boll: Surface } & 1 & Smooth & 309 & 96.56 \\
\hline & & 9 & Pitted & 11 & 3.44 \\
\hline \multirow{2}{*}{22} & \multirow{2}{*}{ Boll: Prominence of tip } & 1 & Blunt & 6 & 1.88 \\
\hline & & 9 & Pointed & 314 & 98.13 \\
\hline 23 & Boll: Opening & 5 & Semi-open & 320 & 100.00 \\
\hline \multirow[t]{4}{*}{24} & \multirow[t]{4}{*}{ Seed: Fuzz } & 1 & Naked & 3 & 0.94 \\
\hline & & 3 & Sparse & 40 & 12.50 \\
\hline & & 5 & Medium & 227 & 70.94 \\
\hline & & 7 & Dense & 50 & 15.63 \\
\hline \multirow[t]{4}{*}{25} & \multirow[t]{4}{*}{ Seed: Fuzz colour } & 1 & White & 20 & 6.25 \\
\hline & & 2 & Grey & 288 & 90.00 \\
\hline & & 3 & Green & 8 & 2.50 \\
\hline & & 4 & Brown & 4 & 1.25 \\
\hline \multirow[t]{4}{*}{26} & \multirow[t]{4}{*}{ Fibre: Colour } & 1 & White & 20 & 6.25 \\
\hline & & 2 & Cream & 293 & 91.56 \\
\hline & & 3 & Green & 4 & 1.25 \\
\hline & & 4 & Brown & 3 & 0.94 \\
\hline
\end{tabular}


Table.2 Coefficient of variation for seed cotton yield and quality traits observed in G. hirsutum cotton germplasm

\begin{tabular}{|l|l|l|l|l|l|l|l|}
\hline \multicolumn{1}{|c|}{ Characters } & Minimum & \multicolumn{1}{|c|}{ Maximum } & \multicolumn{1}{|c|}{ Range } & \multicolumn{1}{|c|}{ Mean } & \multicolumn{1}{c|}{$\begin{array}{c}\text { Standard } \\
\text { deviation }\end{array}$} & $\begin{array}{c}\text { Coefficient of } \\
\text { variation }\end{array}$ \\
\hline Germination (\%) & 62.00 & 100.00 & 38.00 & 84.94 & 8.90 & 10.48 & 79.21 \\
\hline Root length (cm) & 8.11 & 23.67 & 15.56 & 17.24 & 3.43 & 19.89 & 11.76 \\
\hline Shoot length (cm) & 5.65 & 18.97 & 13.32 & 12.80 & 2.24 & 17.52 & 5.03 \\
\hline Vigour index & 1383.80 & 4093.00 & 2709.20 & 2539.70 & 506.40 & 19.94 & 256392.00 \\
\hline $\begin{array}{l}\text { Dry matter of seedling } \\
\text { (g) }\end{array}$ & 0.14 & 0.34 & 0.20 & 0.23 & 0.04 & 15.61 & 0.001 \\
\hline $\begin{array}{l}\text { Number of } \\
\text { sympodia/plant }\end{array}$ & 22.10 & 34.90 & 12.80 & 27.23 & 2.13 & 7.81 & 4.52 \\
\hline Boll number/plant & 16.60 & 26.00 & 9.40 & 20.66 & 1.33 & 6.43 & 1.76 \\
\hline Fibre: Length (mm) & 2.10 & 6.50 & 4.40 & 4.26 & 0.64 & 14.97 & 0.41 \\
\hline Fibre: Strength (g/tex) & 42.00 & 51.00 & 9.00 & 46.33 & 1.53 & 3.29 & 2.33 \\
\hline Fibre: Fineness (mic.) & 3.80 & 7.10 & 3.30 & 5.74 & 0.55 & 9.56 & 0.30 \\
\hline Fibre: Uniformity (\%) & 13.30 & 25.38 & 12.08 & 19.27 & 1.64 & 8.50 & 2.68 \\
\hline Fibre: Elongation (\%) & 14.48 & 20.46 & 5.98 & 18.20 & 0.89 & 4.91 & 0.80 \\
\hline Seed cotton yield/row (g) & 16.00 & 936.00 & 920.00 & 298.50 & 195.10 & 65.37 & 38065.50 \\
\hline $\begin{array}{l}\text { Seed cotton yield/plant } \\
\text { (g) }\end{array}$ & 20.03 & 133.85 & 113.81 & 75.18 & 15.09 & 20.08 & 227.83 \\
\hline
\end{tabular}


Table.3 Pearson correlation coefficients for quantitative traits of G. hirsutum cotton germplasm

\begin{tabular}{|c|c|c|c|c|c|c|c|c|c|c|c|c|c|c|}
\hline & G\% & $\mathbf{R L}$ & SL & VI & DMS & FL & FS & FF & FU & ELG & NS & NBP & SCYR & SCYP \\
\hline G\% & 1.00 & 0.02 & -0.10 & $0.48 * *$ & -0.06 & 0.01 & -0.11 & 0.07 & 0.07 & -0.04 & 0.02 & 0.04 & $0.22 *$ & 0.06 \\
\hline RL & & 1.00 & $0.65^{* *}$ & $0.82 * *$ & -0.01 & -0.11 & 0.01 & 0.00 & 0.12 & $-0.22 * *$ & 0.00 & -0.08 & -0.06 & -0.09 \\
\hline SL & & & 1.00 & $0.69 * *$ & 0.09 & -0.08 & 0.04 & -0.01 & 0.09 & -0.09 & -0.02 & -0.02 & -0.01 & -0.01 \\
\hline VI & & & & 1.00 & -0.02 & -0.09 & -0.03 & 0.02 & 0.10 & $-0.20 * *$ & 0.01 & -0.03 & 0.09 & -0.02 \\
\hline DMS & & & & & 1.00 & $0.34 * *$ & 0.15 & 0.02 & - $0.28 * *$ & $0.24 * *$ & 0.10 & $0.38 * *$ & 0.04 & $0.41 * *$ \\
\hline FL & & & & & & 1.00 & $0.58 * *$ & $-0.33 * *$ & $\begin{array}{l}- \\
0.89 * *\end{array}$ & $0.44 * *$ & $0.32 * *$ & $0.73 * *$ & 0.03 & $0.43 * *$ \\
\hline FS & & & & & & & 1.00 & $-0.52 * *$ & $-\overline{0.50 * *}$ & $0.46^{* *}$ & 0.12 & $0.47 * *$ & -0.11 & 0.15 \\
\hline FF & & & & & & & & 1.00 & $0.42 * *$ & 0.16 & 0.03 & -0.10 & 0.12 & 0.08 \\
\hline $\mathbf{F U}$ & & & & & & & & & 1.00 & $-0.30 * *$ & $-0.26 * *$ & $-0.60 * *$ & -0.02 & $-\overline{0.32 * *}$ \\
\hline ELG & & & & & & & & & & 1.00 & 0.08 & $0.415^{* *}$ & 0.04 & $0.25 *$ \\
\hline NS & & & & & & & & & & & 1.00 & $0.55^{* *}$ & $0.35 * *$ & $0.47 * *$ \\
\hline NBP & & & & & & & & & & & & 1.00 & 0.18 & $0.71 * *$ \\
\hline SCYR & & & & & & & & & & & & & 1.00 & $0.33 * *$ \\
\hline SCYP & & & & & & & & & & & & & & 1.00 \\
\hline
\end{tabular}

*significant at $\mathrm{p}<0.05, * *$ significant at $\mathrm{p}<0.01$

G\% Germination percentage; RL Root length (cm); SL Shoot length (cm); VI Vigour Index; DMS Dry matter of seedlings; FL Fibre Length (mm); FS Fibre Strength(g/tex); FF Fibre Fineness (micronaire); FU Fibre Uniformity(\%); ELG Elongation (mm); NS Number of sympodia/plant; NBP Number of bolls/plant; SCYR Seed cotton yield/row (g); SCYP Single cotton yield/plant (g) 
Table.4 Eigen values, proportion of variability and morpho-agronomic traits that contributed to the first five principal components (PC) of G. hirsutum cotton germplasm

\begin{tabular}{|l|l|l|l|l|l|}
\hline Characters & \multicolumn{1}{|c|}{ PC1 } & \multicolumn{1}{|c|}{ PC2 } & \multicolumn{1}{|c|}{ PC3 } & \multicolumn{1}{c|}{ PC4 } & \multicolumn{1}{c|}{ PC5 } \\
\hline Germination (\%) & -0.017 & 0.183 & -0.246 & -0.559 & -0.573 \\
\hline Root length (cm) & -0.118 & 0.539 & 0.139 & 0.118 & 0.074 \\
\hline Shoot length (cm) & -0.076 & 0.502 & 0.131 & 0.296 & 0.120 \\
\hline Vigour index & -0.107 & 0.595 & -0.001 & -0.131 & -0.191 \\
\hline Dry matter of seedling(g) & 0.229 & 0.086 & -0.082 & 0.415 & -0.126 \\
\hline Number of sympodia/plant & 0.447 & 0.047 & 0.144 & -0.088 & -0.081 \\
\hline Boll number/plant & 0.319 & 0.059 & 0.381 & -0.023 & -0.104 \\
\hline Fibre: Length (mm) & -0.146 & -0.022 & -0.484 & 0.399 & -0.273 \\
\hline Fibre: Strength(g/tex) & -0.402 & -0.025 & -0.199 & 0.185 & 0.004 \\
\hline Fibre: Fineness(micronaire) & 0.273 & -0.089 & -0.024 & 0.327 & -0.511 \\
\hline Fibre: Uniformity(\%) & 0.245 & 0.117 & -0.321 & -0.11 & 0.449 \\
\hline Fibre : Elongation(mm) & 0.433 & 0.115 & -0.137 & 0.039 & 0.051 \\
\hline Seed cotton yield/row(g) & 0.082 & 0.097 & -0.459 & -0.245 & 0.160 \\
\hline Seed cotton yield/plant $(\mathbf{g})$ & 0.321 & 0.107 & -0.352 & 0.120 & 0.129 \\
\hline Eigen value & $\mathbf{4 . 0 4}$ & $\mathbf{2 . 5 0}$ & $\mathbf{1 . 9 1}$ & $\mathbf{1 . 2 5}$ & $\mathbf{1 . 0 6}$ \\
\hline Variability $(\%)$ & $\mathbf{2 8 . 9 0}$ & $\mathbf{1 7 . 9 0}$ & $\mathbf{1 3 . 6 0}$ & $\mathbf{8 . 9 0}$ & $\mathbf{7 . 5 0}$ \\
\hline Cumulative $(\%)$ & $\mathbf{2 8 . 9 0}$ & $\mathbf{4 6 . 7 0}$ & $\mathbf{6 0 . 3 0}$ & $\mathbf{6 9 . 3 0}$ & $\mathbf{7 6 . 8 0}$ \\
\hline
\end{tabular}


Table.5 Average value per cluster for 14 morpho-agronomic traits of 320 cotton germplasm

\begin{tabular}{|c|c|c|c|c|c|c|c|c|c|c|c|c|}
\hline Variable & I & II & III & IV & $\mathbf{V}$ & VI & VII & VII & IX & $\mathbf{X}$ & XI & XII \\
\hline G\% & 84.75 & 90.00 & 81.20 & 87.00 & 72.00 & 85.60 & 94.00 & 96.00 & 98.00 & 100.00 & 98.00 & 100.00 \\
\hline RL & 17.40 & 8.54 & 8.98 & 9.07 & 8.57 & 17.51 & 20.22 & 23.67 & 21.92 & 20.55 & 21.67 & 20.50 \\
\hline SL & 12.78 & 9.02 & 10.07 & 11.35 & 12.75 & 14.09 & 12.78 & 18.97 & 15.78 & 13.66 & 15.02 & 18.00 \\
\hline VI & 2543.47 & 1574.00 & 1548.24 & 1773.86 & 1535.04 & 2700.95 & 3098.28 & 4093.04 & 3696.88 & 3421.00 & 3596.08 & 3861.00 \\
\hline DMS & 0.23 & 0.21 & 0.23 & 0.25 & 0.26 & 0.23 & 0.25 & 0.27 & 0.30 & 0.24 & 0.19 & 0.20 \\
\hline FL & 27.24 & 27.00 & 27.28 & 26.75 & 28.10 & 26.10 & 26.90 & 25.90 & 28.80 & 28.40 & 29.80 & 24.60 \\
\hline FS & 20.68 & 20.70 & 20.06 & 19.75 & 20.00 & 20.36 & 21.30 & 20.30 & 21.30 & 20.70 & 21.50 & 19.30 \\
\hline FF & 4.25 & 4.30 & 4.62 & 4.60 & 4.90 & 4.20 & 4.60 & 4.60 & 3.70 & 3.40 & 4.00 & 4.20 \\
\hline FU & 46.32 & 46.00 & 46.2 & 46.50 & 46.00 & 47.40 & 47.00 & 47.00 & 45.00 & 45.00 & 45.00 & 48.00 \\
\hline ELG & 5.74 & 6.00 & 6.12 & 6.35 & 5.90 & 5.28 & 6.40 & 5.50 & 5.30 & 5.40 & 5.50 & 5.70 \\
\hline NS & 19.22 & 19.20 & 19.94 & 19.56 & 19.64 & 21.09 & 21.27 & 19.75 & 18.43 & 19.18 & 20.45 & 17.10 \\
\hline NBP & 18.18 & 18.53 & 18.84 & 18.58 & 18.37 & 18.16 & 18.54 & 18.06 & 18.56 & 18.92 & 19.51 & 17.10 \\
\hline SCYR & 273.69 & 398.00 & 586.40 & 789.50 & 789.00 & 807.80 & 762.00 & 769.00 & 303.00 & 936.00 & 632.00 & 264.00 \\
\hline SCYP & 74.35 & 88.77 & 94.93 & 91.14 & 78.43 & 87.11 & 70.45 & 76.57 & 81.47 & 86.08 & 108.48 & 71.40 \\
\hline
\end{tabular}

Table.6 Distance between 12 clusters of 320 cotton germplasm

\begin{tabular}{|c|c|c|c|c|c|c|c|c|c|c|c|c|}
\hline Cluster & I & II & III & IV & V & VI & VII & VII & IX & $\mathbf{X}$ & XI & XII \\
\hline I & & 977.58 & 1043.45 & 926.67 & 1132.58 & 557.00 & 739.17 & 1626.87 & 1153.89 & 1099.59 & 1112.54 & 1317.00 \\
\hline II & & & 190.46 & 439.59 & 393.5 & 1199.20 & 1567.30 & 2546.31 & 2125.09 & 1923.83 & 2035.74 & 2291.00 \\
\hline III & & & & 303.65 & 203.93 & 1173.86 & 1560.24 & 2551.51 & 2167.40 & 1905.26 & 2048.51 & 2335.00 \\
\hline IV & & & & & 239.64 & 927.33 & 1324.94 & 2319.39 & 1983.71 & 1653.75 & 1829.18 & 2152.00 \\
\hline $\mathbf{V}$ & & & & & & 1166.21 & 1563.69 & 2558.24 & 2215.99 & 1891.94 & 2067.44 & 2384.00 \\
\hline VI & & & & & & & 400.41 & 1392.73 & 1116.66 & 731.53 & 912.58 & 1281.00 \\
\hline VII & & & & & & & & 994.83 & 754.43 & 367.04 & 515.94 & 910.00 \\
\hline VIII & & & & & & & & & 611.68 & 692.59 & 516.53 & 555.00 \\
\hline IX & & & & & & & & & & 690.53 & 345.16 & 169.00 \\
\hline $\mathbf{X}$ & & & & & & & & & & & 351.54 & 803.00 \\
\hline XI & & & & & & & & & & & & 455.00 \\
\hline XII & & & & & & & & & & & & \\
\hline
\end{tabular}


Fig.1 Dendrogram showing the clustering of qualitative traits in G. hirsutum cotton germplasm

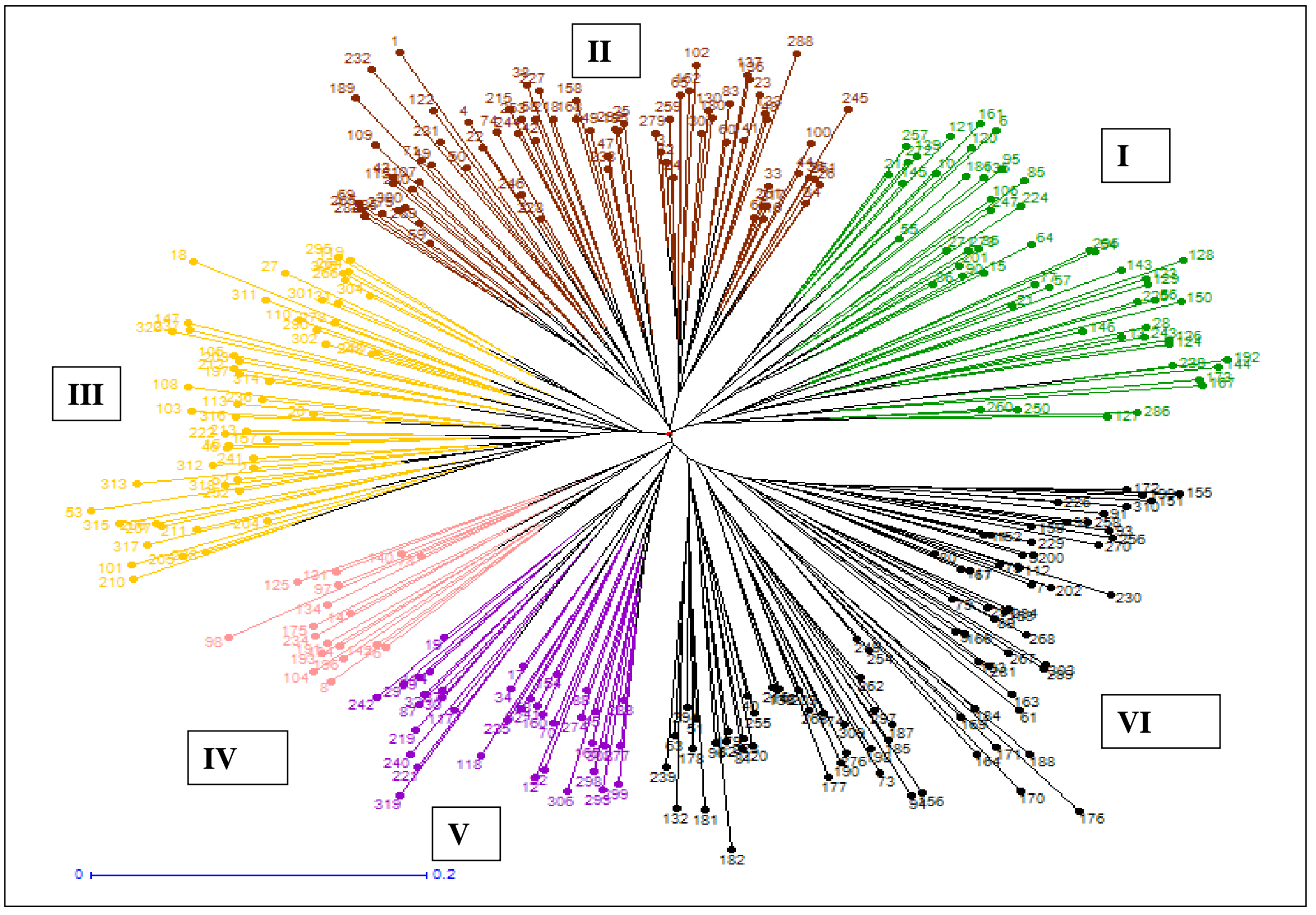


Fig.2 Biplot of Principal Component 1 and 2 of G. hirsutum cotton germplasm



Fig.3 Loading biplot of Principal Component 1 and 2 of G. hirsutum cotton germplasm

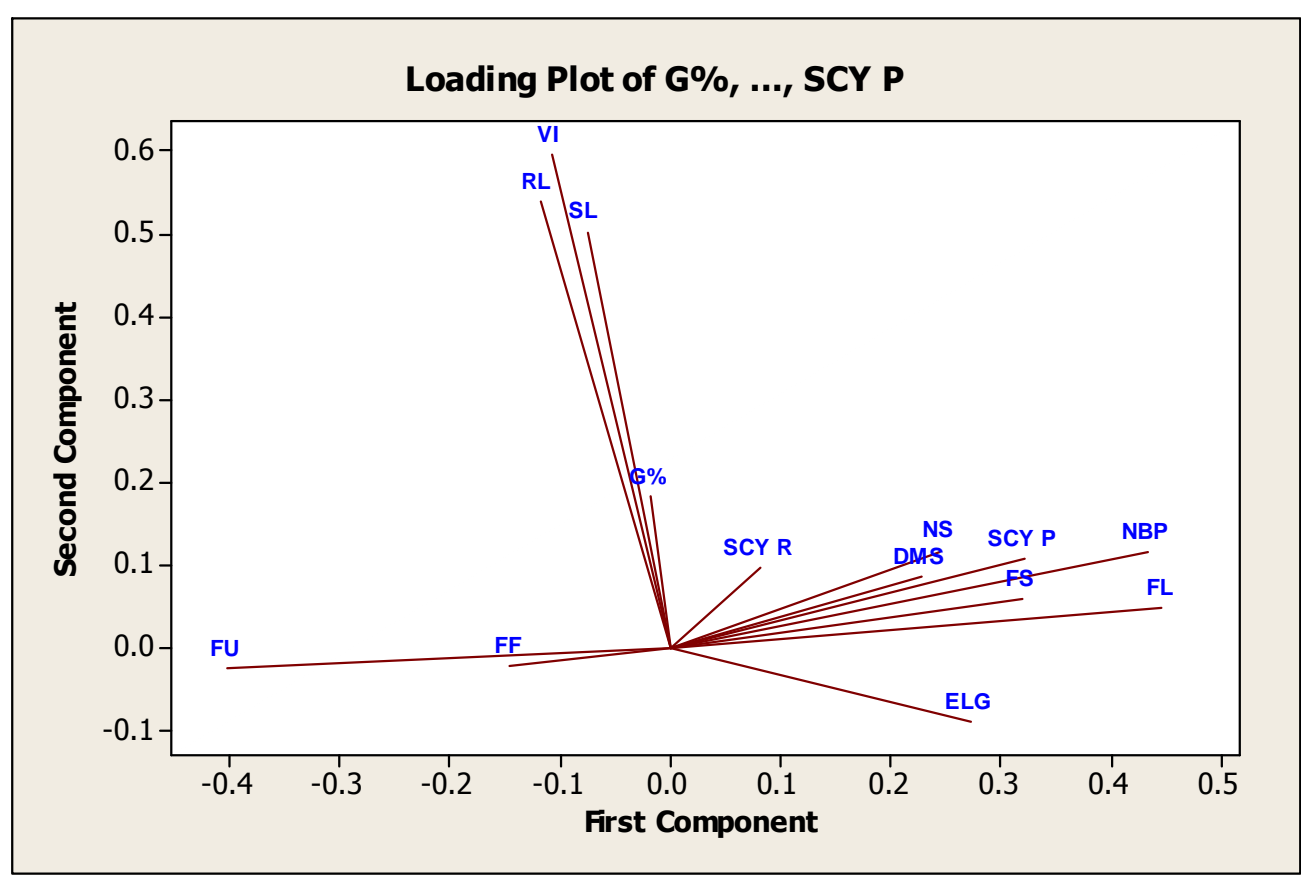


Fig.4 Two dimensional ordinates of principal components of G. hirsutum cotton germplasm

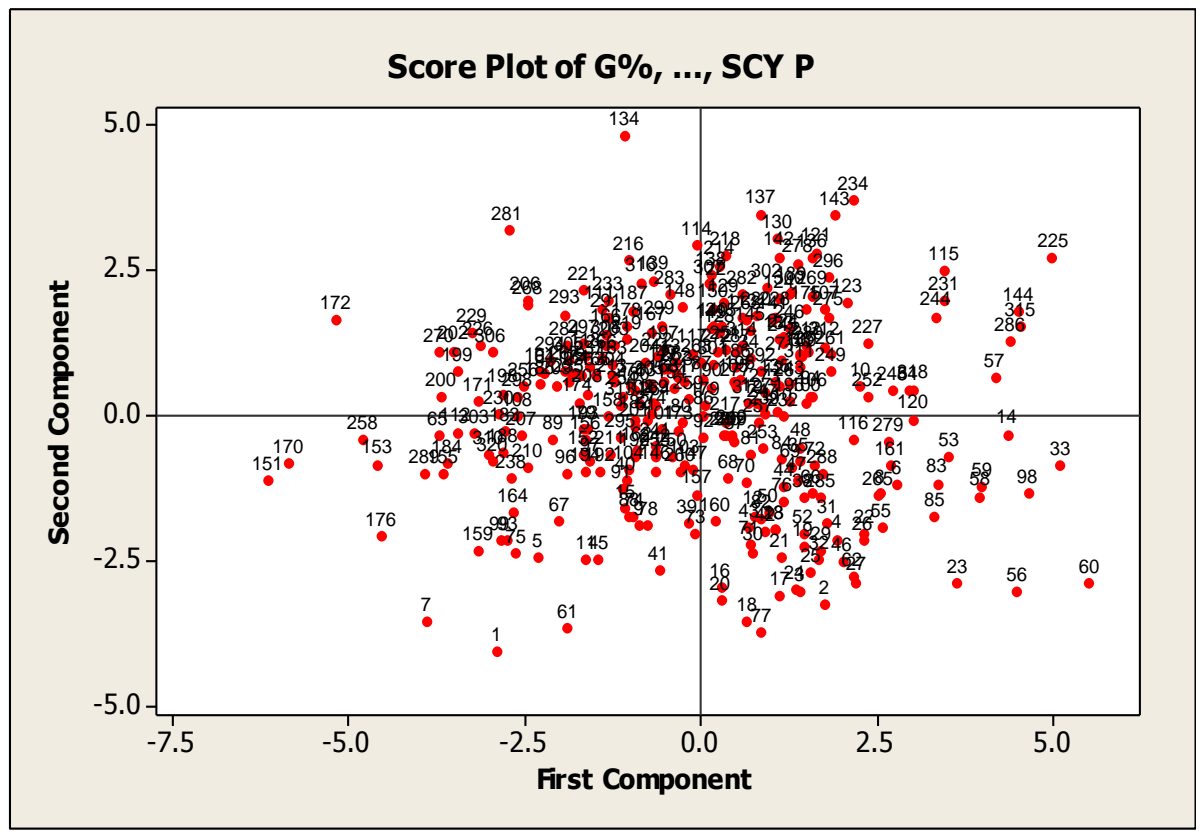

Fig.5 Scree plot showing the Eigen values of different principal components of cotton germplasm

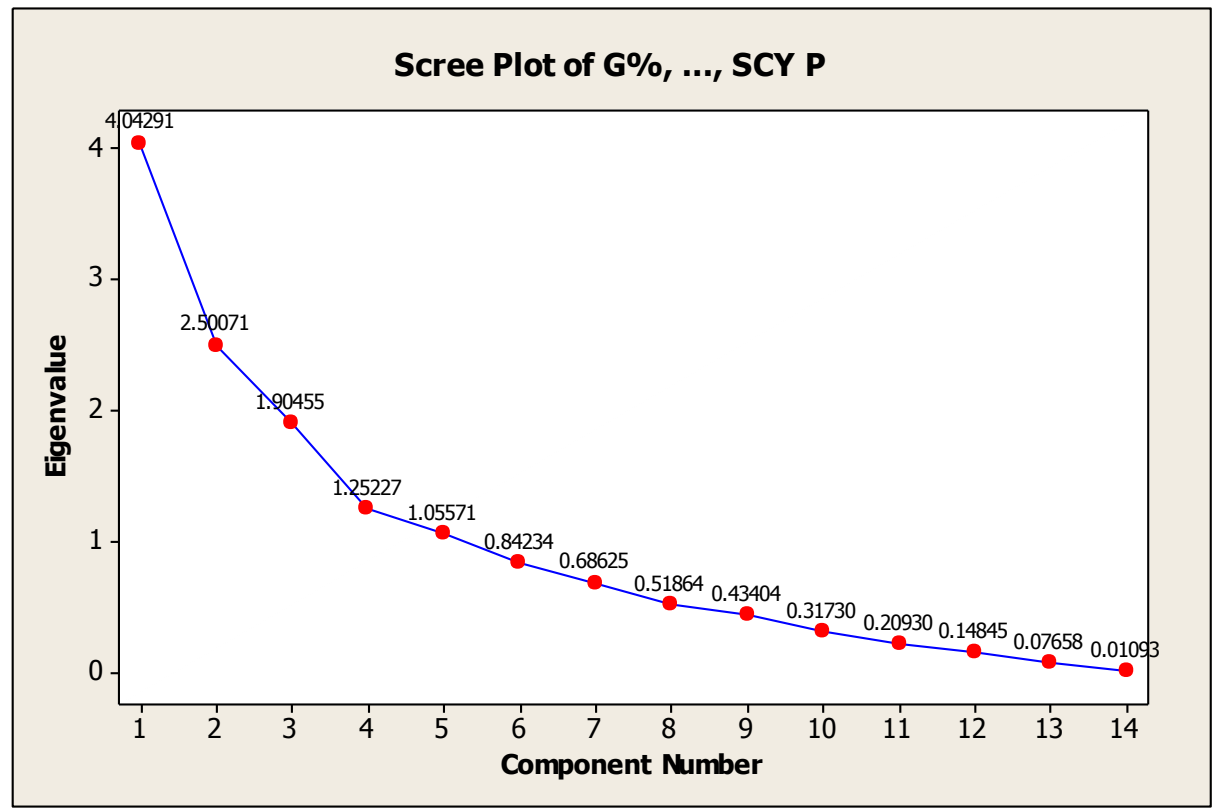


Fig.6 Dendrogram showing the clustering of quantitative traits in G. hirsutum cotton germplasm

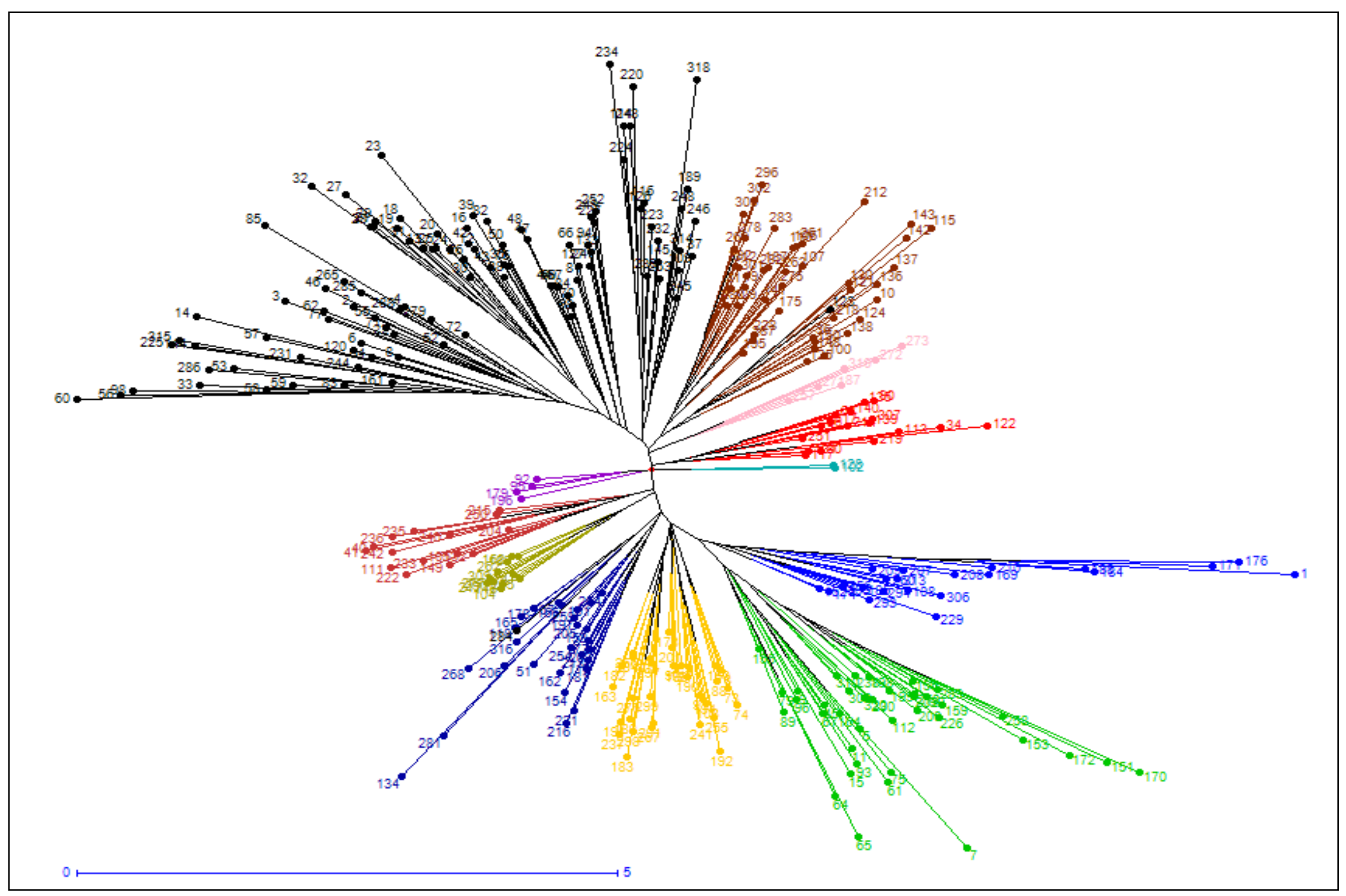




\section{Clustering}

The factors corresponding to five PCs were subjected to cluster analysis based on Euclidean distances and grouped by unweighted paired group method using the arithmetic average (UPGMA) using DARwin 6 . The dendrogram depicted twelve distinct clusters is presented in (Fig. 6). The cluster $\mathrm{V}$ was the largest followed by cluster IV, XI, X, IX, XII, II, VII, III, VIII, VI and I. May et al., (1995) reported that cluster analysis identified groups of cotton cultivars those were more closely related. These results are in confirmation with the earlier studies on Gopinath et al., (2009) and Satish et al., (2009). The geographical distribution of genotypes is not the only factor that causes morphological and genetic diversity. Genetic diversity may be due to the outcome of several other factors like natural and forced selection, exchange of breeding material, genetic drift and environmental variation. Therefore, selection of parents for crop improvement programme should be based on genetic rather than geographical diversity.

Cluster analysis using unweighted paired group method using arithmetic average showed the distinct pattern of group formation (Table 5). The genotypes in cluster III showed higher values of seed cotton yield per plant. The cluster $\mathrm{V}$ is contributed mainly by fibre elongation and the number of sympodia. The cluster VIII is contributed by the dry matter of seedlings, cluster $\mathrm{X}$ by germination percentage and seed cotton yield per row. Cluster XI was characterised by higher values of fibre length, fibre strength and the number of bolls per plant. Cluster XII was comprised of genotypes having the highest and reasonable values of germination percentage and fibre uniformity.

The inter-cluster distance (Table 6) was maximum in between cluster $\mathrm{V}$ and VIII
(2558.24) and minimum in between cluster $X$ and XII (169). The range of inter-cluster values ranged from 169 to 2558 indicates the wide range of diversity. Choosing of genotypes belonging to distant clusters was expected to exploit maximum heterosis in hybrids. These distant genotypes may also be used for the synthesis of the wide spectrum of variation among the segregating population.

In conclusion, based on the results of the present investigation, an extensive range of genetic diversity has been explored in cotton accessions. The traits like the number of bolls per plant, the number of sympodia per plant, seed cotton yield per row showed significant positive association with seed cotton yield per plant which served as a criterion to select promising cotton genotypes. The traits like fibre length, the number of bolls per plant, seed cotton yield per plant, fibre elongation and fibre strength showed high variability in biplot analysis which in accordance with the correlation analysis. So these traits are very much useful for cotton improvement programme. The principal component analysis helped in the identification of diversified cotton genotype and these genotypes might be utilised in the breeding program.

\section{Acknowledgement}

The author profusely thanks, Dr. Punit Mohan, Principal Scientist, CICR, Nagpur for the supply of seeds of germplasm accessions. I sincerely acknowledge the Protection of Plant Varieties and Farmers Rights and Technology Mission on Cotton for generous financial support.

\section{References}

Ahmad MQ, SH Khan and FM Azhar (2012) "Decreasing Level of Genetic Diversity in Germplasm and Cultivars of Upland 
Cotton (Gossypium hirsutum) in Pakistan." J. Agric. Soc. Sci., 8(3): 9296.

Ahsan A, A Naeem, A Muhammad, A Muhammad, T Asif, A Rashid, E Muhammad and A Salman (2011) Comparative performance of $\mathrm{Bt}$ cotton with some elite conventional cotton cultivars under arid to semi-arid conditions. Afr. J. Agric. Res., 6: 16001606.

Anon (2016) cotcorp.gov.in >National Cotton Scenario)

Anon (2016) www.cottoninc.com/ corporate/../ World-Cotton-ProductionExp.pdf.

Bibi, M, N Khan, F Mohammad, R Gul, A Khakwani, O Sayal, I Khan and M Idrees (2011) Genetic disparity and relationship among quantitatively inherited yield related traits in diallel crosses of upland cotton. Pak. J. Bot. 43: 2543-2550.

Brown-Guedira, G, J Thompson, R Nelson and M Warburton (2000) Evaluation of genetic diversity of soybean introductions and North American ancestors using RAPD and SSR markers. Crop Sci 40: 815-823.

Esmail, RM, JF Zhang and AM Abdel-Hamid (2008) Genetic diversity in elite cotton germplasm lines using field performance and RAPD markers. World Journal of Agriculture Science 4: 369375.

Gopinath M, S Rajamani, RK Naik and CHM Rao (2009) Genetic divergence for lint characters for upland cotton (G. hirusutum L.). J. Cotton Res. Dev. 23: $18-22$.

Hussain, K, I Khan, H Sadaqat and M Amjad (2010) Genotypic and phenotypic correlation analysis of yield and fiber quality determining traits in upland cotton (Gossypim hirsutum). Int. J. Agric. Biol., 12: 348-352.
Khan FUZ, SU Rehman, MA Abid, W Malik, CM Hanif, $M$ Bilal and $G$ Qanmber (2015) Exploitation of germplasm for plant yield improvement in cotton (Gossypium hirsutum L.). J. Green Physiol. Genet. Genom., 1(1): 1-10.

Khan, NU and G Hassan (2011) Genetic effects on morphological and yield traits in cotton (Gossypium hirsutum L.). Spanish J. Agric. Res., 9(2): 460-472.

Li, Z, X Wang, Z Yan, Z Guiyin, L Wu, C Jina and Z MA (2008) Assessment of genetic diversity in glandless cotton germplasm resources by using agronomic traits and molecular markers. Frontiers of Agriculture in China 2: 245-252.

May, OL, DT Bowman and DS Calhoun (1995) Genetic diversity of US Upland cotton cultivars released between 1980 and 1990. Crop Sci., 35: 1570-1574.

Raji, AA (2002) Assessment of genetic diversity and heterotic relationships in African improved and local cassava (Manihet esculenta Crantz) germplasm. Ph.D. thesis. University of Ibadan, Nigeria.

Sajjad, M, S Khan and A Khan (2011) Exploitation of germplasm for grain yield improvement in spring wheat (Triticum aestivum). Int. J. Agric. Biol. 13: 695-700.

Salahuddin, S, S Abro, A Rehman and K Iqbal (2010) Correlation analysis of seed cotton yield with some quantitative traits in upland cotton (Gossypium hirsutum L.). Pak. J. Bot., 42(6): 37993805.

Sanni, KA, I Fawole, RG Guei, DK Ojo and EA Somado (2008) Geographical patterns of phenotypic diversity in Oryza sativa landraces of Côte d'Ivoire, Euphytica, 160: 389-400.

Saravanan, S, P Arutchendhil, TS Raveendran and K Koodalimgam (2006) Assessment of genetic divergence 
among introgressed culture of Gossypium hirsutum L. through RAPD analysis. J. Applied Sci., Res., 2: 12121216.

Satish, Y, PP Jain, and BS Chhabra (2009) Studies on genetic diversity in upland cotton (G. hirsutum L.). J. Cotton Res. Development, 23(1): 18-22.

Shahzad, MT, F Ijaz, O Khan, B Saleem and U Hassan (2015) Correlation, Path Analysis \& Heritability Among Some Yield and Fibre Related Traits of Gossypium hirsutum L. Cotton Genomics and Genet., 6 (4): 1-7.

Shakeel, A, I Talib, M Rashid, A Saeed, K Ziaf and MF Saleem (2015) Genetic diversity among upland cotton genotypes for quality and yield related traits. Pak. J. Agri. Sci., 52(1): 73-77.

Soomro, Z, A Larik, M Kumbhar, N Khan and N Panhwar (2008) Correlation and path analysis in hybrid cotton. Sabro J.
Breed. Genet., 40: 49-56.

Sundar, R, SS Patil, H Ranganath, P Srivalli, S Manjula and K Pranesh (2014) Genetic Diversity Analysis in Cotton (Gossypium hirsutum L.) based on morphological traits and microsatellite markers. Int. J. Basic and Applied Biol., 1(1): 19-22.

Taohua, Z and C Yichun (1993) Canonical correlation and principal component analysis among agronomic traits and yield in cotton ( $G$. hirsutum L.) population. J. Anhui Agric. Uni. 2: 008 pages.

Ward, JH (1963) Hierarchical grouping to optimize an objective function. $\mathrm{J}$. American Statistical Association, 58(301): 236-244.

Yan W and I Rajcan (2002) Biplot Analysis of test Sites and Trait relations of Soybean in Ontario. Crop Sci., 42: 1120.

\section{How to cite this article:}

Rathinavel, K. 2019. Agro-morphological Characterization and Genetic Diversity Analysis of Cotton Germplasm (Gossypium hirsutum L.). Int.J.Curr.Microbiol.App.Sci. 8(02): 2039-2057. doi: https://doi.org/10.20546/ijcmas.2019.802.237 